\title{
A study comparing VVI and DDI pacing in elderly patients with carotid sinus syndrome
}

\author{
Shona J McIntosh, Joanna Lawson, Rodney S Bexton, Ronald G Gold,
} Margaret M Tynan, Rose Anne Kenny

\begin{abstract}
Objective-To determine whether single chamber ventricular demand (VVI) pacing is adequate for elderly patients with carotid sinus syndrome.

Design-Prospective double blind randomised cross over study.

Setting-Tertiary referral centre

Patients-30 consecutive patients aged over 60 years with carotid sinus syndrome referred for cardiac pacing.

Intervention-Patients underwent dual chamber pacemaker implantation and were then randomised to two threemonth periods of VVI and DDI pacing.

Main outcome measures-Responses to cardiovascular tests (vasodepression during carotid sinus massage, pacemaker effect, postural blood pressure measurements, and response to head up tilt), and symptoms.
\end{abstract}

Results-11 patients developed profound hypotension during upright carotid sinus massage while pacing VVI compared with only two while pacing DDI. The upright pacemaker effect was greater in VVI (VVI, -31 (SD 19) $\mathrm{mm}$ Hg $v$ DDI, -4 (12) $\mathrm{mm}$ Hg; $P<0.001)$. Postural blood pressure measurements and responses to head up tilt did not vary. Eleven patients were unable to tolerate VVI pacing and had to be withdrawn early from this limb of the study (group A). Fourteen of the remainder completed diary cards and did not express a preference (group B). No patient preferred VVI. Group A patients were older (group A, 78 (6) years $v$ group $B, 70$ (9) years; $P<0.05$ ), were more likely to be female (group A, $73 \% v$ group B, $14 \% ; P<0.01$ ), and were more likely to have orthostatic hypotension while pacing DDI (group A, $46 \% v$ group B, $0 \% ; P<$ $0 \cdot 01)$. Group $A$ and $B$ patients could not be differentiated by other prepacing clinical or haemodynamic variables.

Conclusions-Elderly patients with carotid sinus syndrome are likely to develop symptomatic hypotension following VVI pacing. The optimum pacing mode for individual patients cannot be predicted by simple cardiovascular tests before pacing.

(Heart 1997;77:553-557)

Keywords: carotid sinus syndrome; elderly; pacing
Carotid sinus syndrome is an important, but frequently overlooked, cause of syncope and presyncope in the elderly. ${ }^{1}$ The condition is diagnosed in a patient with otherwise unexplained symptoms when five seconds of carotid sinus massage produces either asystole exceeding three seconds (cardioinhibitory subtype), a fall in systolic blood pressure exceeding $50 \mathrm{~mm} \mathrm{Hg}$ in the absence of significant cardioinhibition (vasodepressor subtype), or a combination of the two (mixed subtype). ${ }^{23}$

Cardiac pacing abolishes syncope in up to $90 \%$ of patients with cardioinhibition. ${ }^{45} \mathrm{~A}$ significant number, however, have residual presyncopal symptoms, notably those receiving a single chamber ventricular system. Such residual symptoms have been attributed to the development of pacemaker syndrome and to the adverse effect of ventricular pacing on cardiac haemodynamics, both of which may result in increased vasodepression. ${ }^{6}$

Dual chamber cardiac pacing protects against pacemaker syndrome while maintaining more physiological cardiac haemodynamics ${ }^{7-9}$ but is both time consuming and costly. ${ }^{10}$ Although it is now recommended that dual chamber pacing systems are used in all patients with carotid sinus syndrome, elderly patients with this condition still often undergo cheaper ventricular pacing on the assumption that this will be adequate for older patients with a more sedentary lifestyle. ${ }^{1112}$

The aim of this study was to compare the haemodynamic effects of single chamber ventricular demand (VVI) pacing with those of dual chamber demand (DDI) pacing in elderly patients with carotid sinus syndrome in order to determine whether VVI pacing is adequate for elderly subjects with this condition. A secondary aim was to evaluate clinical and haemodynamic variables which might predict the choice of pacing mode prepacing.

\section{Methods}

The study population comprised a consecutive series of 30 elderly patients with carotid sinus syndrome referred from a "syncope" clinic for cardiac pacing. Study patients had recurrent syncope, presyncope, or falls. All had undergone a standardised investigative protocol at the syncope clinic incorporating a detailed clinical assessment, a 12 lead surface electrocardiogram, a minimum of 24 hours of Holter monitoring, and cardiovascular testing. All patients had reproducible symptomatic cardioinhibitory or mixed carotid sinus syndrome 
and this was the only attributable cause of symptoms.

Study patients underwent dual chamber cardiac pacing (Chorus II; ELA) and were then randomised to two three-month periods of ventricular (VVI) and dual chamber (DDI) pacing. (Two six-week periods were used for the final 10 patients.) The basic pacing rate in both modes was $80 / \mathrm{min}$; in DDI mode $25 \%$ hysteresis was used. All patients were assessed for the presence of retrograde atrioventricular conduction at the time of pacing by recording the atrial electrocardiogram during ventricular pacing at $80-120$ beats/min.

Cardiovascular testing was repeated in each pacing mode; the change in blood pressure with the onset of pacing (pacemaker effect) was also assessed. The study was conducted in a double blind manner with neither patients nor cardiovascular investigators aware of the pacing mode. Patients completed daily symptom diaries throughout the study, noting the presence or absence of syncopal episodes, presyncopal episodes, or falls on each day. All subjects gave informed consent and the study was approved by the Newcastle ethics committee.

\section{CARDIOVASCULAR TESTING}

Heart rate and phasic blood pressure were recorded non-invasively using a surface electrocardiogram (paper speed $25 \mathrm{~mm} / \mathrm{s}$ ) and Finapres (digital photoplethysmography) respectively.

\section{Carotid sinus massage}

Patients were tested both supine and upright $\left(70^{\circ}\right.$ head up tilt). Five seconds of longitudinal massage was applied over the point of maximum carotid impulse. This was repeated on right and left sides allowing a 30 second interval between tests. Continuous heart rate and blood pressure measurements were made. Prepacing the vasodepressor response independently of cardioinhibition was assessed in those with more than 1.5 seconds asystole by repeating massage following the administration of $600 \mu \mathrm{g}$ intravenous atropine. Postpacing the vasodepressor response independently of pacemaker effect was assessed by performing massage during fixed rate pacing (achieved using a magnet).

\section{Postural blood pressure measurements}

Blood pressure was recorded after 20 minutes supine rest and at 30 seconds and one minute after standing unsupported.

\section{Head up tilt}

Patients were tilted head up at $70^{\circ}$ (Akron automated tilt table: foot plate support) for 30 minutes. Blood pressure was recorded continuously.

\section{Pacemaker effect (postpacing)}

The change in blood pressure with the onset of pacing (induced using a magnet) was assessed supine and during $70^{\circ}$ head up tilt.

\section{DIAGNOSTIC CRITERIA}

These were based on published data.

\section{Carotid sinus syndrome}

Carotid sinus massage producing asystole exceeding three seconds (cardioinhibitory subtype), a fall in systolic blood pressure exceeding $50 \mathrm{~mm} \mathrm{Hg}$ in the absence of significant cardioinhibition (vasodepressor subtype), or both responses (mixed subtype). ${ }^{23}$

\section{Vasovagal syncope}

Symptomatic hypotension with or without bradycardia induced by head up tilt. ${ }^{13}$

\section{Orthostatic hypotension}

A fall in systolic blood pressure exceeding $20 \mathrm{~mm} \mathrm{Hg}$ within one minute of standing unsupported. ${ }^{13}$

\section{STATISTICAL ANALYSIS}

Parametric data was compared using Student's paired $t$ test. Symptom scores were assessed using the Fisher exact test. Non-parametric data was analysed using the MannWhitney $U$ test. Values are given as mean (SD).

\section{Results}

\section{CLINICAL CHARACTERISTICS}

The study group comprised 30 patients with carotid sinus syndrome, mean (SD) age 79 (9) years (17 male, 13 female), referred for cardiac pacing between January 1992 and January 1994. Presenting complaints were dizziness and syncope $(47 \%)$, syncope $(20 \%)$, dizziness and falls $(13 \%)$, dizziness $(13 \%)$, and all three symptoms $(7 \%)$. The median duration of symptoms was 20 months (range 1 to 240 ). Twenty three patients were diagnosed as having cardioinhibitory carotid sinus syndrome on initial testing; the remainder had a mixed response to carotid sinus massage. Seventy three per cent of patients showed retrograde atrioventricular conduction at the time of pacing.

\section{RESPONSES TO CARDIOVASCULAR TESTS}

PREPACING AND IN VVI AND DDI PACING MODES Eleven patients developed profound hypotension during upright carotid sinus massage while pacing VVI and were returned to the supine position before the exact fall in blood pressure could be documented. Only two patients developed symptomatic hypotension during upright massage when pacing DDI. There were, however, insufficient data to allow a comparison of upright vasodepressor responses, and supine measurements were used. The pacemaker effect obtained in the upright position was used for data analysis.

Supine vasodepression in VVI mode was similar to that prepacing, while the response in DDI was less than prepacing $(P<0.01$; table 1 ). The pacemaker effect in VVI was greater than that in DDI $(P<0.001)$. Responses to active standing and head up tilt did not vary.

\section{SYMPTOM SCORES}

Eleven patients were unable to tolerate VVI pacing and had to be withdrawn prematurely, at a median of seven days, from this limb of the study (group A). Symptoms were dizziness 
Table 1 Cardiovascular test results prepacing and in VVI and DDI pacing modes

\begin{tabular}{lcccl}
\hline Response & Prepacing & \multicolumn{1}{l}{ VVI } & DDI & Significance \\
\hline Vasodepression (mm Hg) & $33(16)^{\star}$ & $27(15)$ & $22(11)$ & $\star \mathrm{P}<0.01$ \\
Postural BP change (mm Hg) & $-12(24)$ & $-5(22)$ & $-7(18)$ & NS \\
Orthostatic hypotension (\%) & $\mathbf{4 4}$ & 22 & 20 & NS \\
Pacemaker effect (mm Hg) & $-31(19) \dagger$ & $-4(12)$ & $\dagger \mathrm{P}<0.001$ \\
Vasovagal (\%) & 14 & 12 & 7 & NS \\
\hline
\end{tabular}

$\mathrm{BP}$, blood pressure.

$\star \mathrm{P}<0.01$ prepacing $v$ DDI; $\dagger \mathrm{P}<0.001 \mathrm{VVI} v \mathrm{DDI}$

Table 2 Cardiovascular test results in groups $A$ and $B$

\begin{tabular}{lccl}
\hline Response & Group $A$ & Group B & Significance \\
\hline Cardioinhibition (s) & $5 \cdot 2(1 \cdot 7)$ & $6 \cdot 3(1 \cdot 3)$ & NS \\
RAVC (\%) & 82 & 64 & NS \\
Vasodepression (mm Hg) & $24(12)$ & $38(17)$ & NS \\
Prepacing & $24(9)$ & $29(17)$ & NS \\
VVI & $21(12)$ & $24(10)$ & NS \\
DDI & $-14(22)$ & $-10(29)$ & NS \\
Postural BP change (mm Hg) & $-8(25)$ & $-6(17)$ & NS \\
Prepacing & $-16(20)$ & $-2(12)$ & P $<0.05$ \\
VVI & 40 & 50 & NS \\
DDI & 20 & 29 & NS \\
Orthostatic hypotension (\%) & 46 & 0 & P $<0.01$ \\
Prepacing & $-35(21)$ & $-21(19)$ & NS \\
VVI & $-4(8)$ & $-4(15)$ & NS \\
DDI & &
\end{tabular}

RAVC, retrograde atrioventricular conduction; $\mathrm{BP}$, blood pressure.

(six patients), dizziness and syncope (one), falls (one), and unspecified (three). Only four group A patients completed diary cards; all reported significantly more symptoms while pacing VVI. Fifty five per cent of patients in group $\mathrm{A}$ had an unrecordable blood pressure during upright carotid sinus massage while pacing VVI. The remaining patients completed both limbs of the study and did not express a preference for either pacing mode. Fourteen of these patients completed diary cards. The only symptom reported by this group was dizziness, but symptom scores for VVI and DDI were similar (group B). No patient preferred VVI.

Compared with group B, patients in group A were older $(78(6)$ years $v 70$ (9) years; $\mathrm{P}<$ $0.05)$ and were more likely to be female $(73 \% v$ $14 \% ; P<0.01)$. Cardioactive drug use was similar in the two groups $(55 \% v 50 \%)$, as was initial cardioinhibition and the frequency of retrograde atrioventricular conduction (table 2 ). The frequency of orthostatic hypotension and the degree of orthostatic blood pressure change while pacing DDI were greater in group A. Cardiovascular test results prepacing and in each pacing mode were, otherwise, similar for the two groups (table 2).

COMPLICATIONS OF CAROTID SINUS MASSAGE Two patients developed transient neurological symptoms and signs following carotid sinus massage. Both made a full recovery.

\section{Discussion}

Carotid sinus syndrome is an important but frequently overlooked cause of syncope and presyncope, particularly in the elderly. ${ }^{1}$ In centres which routinely perform carotid sinus massage it is diagnosed in up to $40 \%$ of older individuals with recurrent unexplained symptoms. ${ }^{13}$ Cardioinhibitory and mixed subtypes of this condition are treated with cardiac pacing. Such treatment is currently recommended in individuals who have experienced two or more syncopal episodes (though some authorities advise intervention after a solitary syncopal attack). ${ }^{1}$ The pacing rate for carotid sinus syndrome in the United Kingdom, however, remains low, with figures ranging between $1.5 \%$ and $21 \%$ depending upon the region studied. ${ }^{14}$ This suggests that the condition is underdiagnosed or undertreated, or both. Of note in this regard is the fact that up to $30 \%$ of older patients with carotid sinus syndrome have amnesia for loss of consciousness and present only with unexplained falls. ${ }^{15}$

The choice of pacing mode in carotid sinus syndrome has, for many years, been a controversial issue. Atrial pacing is contraindicated since the majority of these patients develop atrioventricular block in addition to sinus arrest during carotid sinus reflex activation. ${ }^{4}$ Ventricular pacing abolishes cardioinhibition but a significant number of individuals are left with residual hypotensive symptoms which have been attributed to the development of pacemaker syndrome and to loss of atrioventricular synchrony, both of which may exacerbate vasodepression. ${ }^{6}$

Pacemaker syndrome occurs with ventricular pacing when retrograde ventriculoatrial conduction is intact - a condition which exists during reflex activation in up to $80 \%$ of patients with carotid sinus syndrome. ${ }^{4}$ The contraction of retrogradely activated atria against closed atrioventricular valves results in reduced ventricular filling (as atrial blood is returned to the pulmonary and systemic veins) and possibly in reflex vasodilatation (as a result of raised intraatrial pressure). In consequence, there is a fall in blood pressure with the onset of pacing. Even in the absence of retrograde conduction, ventricular pacing may produce a significant fall in cardiac output as the atrial contribution to ventricular filling is lost. ${ }^{7}$ This may be particularly marked in elderly individuals, in whom diastolic filling is already impaired. ${ }^{16}$

Dual chamber cardiac pacing maintains atrioventricular synchrony and (except in rare circumstances) protects against pacemaker syndrome. Indeed, the superior haemodynamic effects of this form of cardiac pacing have long been recognised. ${ }^{89}$ However, although the British Pacing and Electrophysiology Group (BPEG) has recommended DDI as the pacing mode of choice in patients with carotid sinus syndrome, many argue that such complex and expensive systems are unnecessary in elderly and more sedentary individuals. ${ }^{11} 12$ Indeed, recent figures suggest that although patients over 75 years account for more than $60 \%$ of pacemaker implants in the United Kingdom, only a small minority of these patients receive a dual chamber system. ${ }^{17}$ The equivalent figure for elderly patients with carotid sinus syndrome is not known since this condition accounts for less than $2 \%$ of pacemaker implants nationally. ${ }^{18}$ It is, however, likely to be similar.

Increased recognition of carotid sinus syndrome as an attributable cause of syncope and unexplained falls, coupled with the growing 
practice of active intervention in older individuals, should increase the demand for pacing in carotid sinus syndrome. However, an increased use of dual chamber pacing systems in older patients has enormous health care and cost implications. Dual chamber pacing systems cost $40-100 \%$ more than ventricular systems, require more technical expertise to implant, and require more specialised (often regional centre) follow up. ${ }^{11} \mathrm{~A}$ recent report by the Yorkshire College and Centre for Health Services Research, based on a review of recently published reports, has raised serious doubts about the cost-effectiveness of using such complex pacing systems. ${ }^{19}$ The report emphasises that although pacing improves quality of life in carotid sinus syndrome, there is no evidence that it influences survival. Additionally, it points out that the BPEG recommendations for complex systems in this and other bradyarrhythmias are based on observational data and not on the results of prospective randomised trials in defined patient groups.

In the present study dual chamber pacing was shown to offer clear haemodynamic advantages over single chamber ventricular pacing in elderly patients with carotid sinus syndrome. The fall in blood pressure with the onset of pacing (or pacemaker effect) was significantly greater in VVI than in DDI, reflecting the fact that - in keeping with other series ${ }^{4}$ $73 \%$ of these patients had intact retrograde atrioventricular conduction.

The vasodepressor response to carotid sinus massage in DDI mode was significantly less than that elicited prepacing. This was because pacemakers were programmed with $60 / 80$ hysteresis, resulting in higher heart rates during carotid sinus massage after pacing than before. Additionally, only patients with asystole exceeding 1.5 seconds prepacing were given atropine. Although vasodepressor responses in the two pacing modes were similar when assessed supine, 11 patients developed symptomatic hypotension during upright carotid sinus massage while pacing VVI and this precluded measurement of the upright vasodepressor response in these individuals. Had these data been available it is likely that upright vasodepression in VVI would have been greater than in DDI. Indeed, in a similar study comparing VVI and DVI pacing modes, Madigan et al showed that vasodepression was greatest during upright carotid sinus massage while pacing VVI, and also observed that patients had more symptoms during this procedure when pacing VVI. ${ }^{8}$

Eleven patients were unable to tolerate VVI pacing. In the present study these symptomatic individuals could not be distinguished from asymptomatic subjects by the presence of retrograde atrioventricular conduction or by prepacing cardiovascular tests. In similar randomised studies, prepacing predictors for failure of VVI pacing have included the presence of retrograde atrioventricular conduction, orthostatic hypotension, and an increased and symptomatic pacemaker effect (during temporary VVI compared with temporary DVI pac- ing). ${ }^{20}$ Indeed, Brignole et al selected 34 patients without these criteria (and without a symptomatic vasodepressor response) for ventricular pacing. At a mean (SD) of 32 (10) months follow up, symptoms in this group were similar to those in 26 patients with these hypotensive features who had undergone dual chamber cardiac pacing. ${ }^{21}$ The author concluded that selected patients with carotid sinus syndrome can be treated with a single chamber ventricular system and that these patients can be identified prepacing. In the present study the pacemaker effect prepacing was not assessed. Routinely performing temporary pacing before permanent pacing in order to assess this would, however, be both time consuming and costly.

This study goes some way to support the BPEG recommendations for complex systems in carotid sinus syndrome. One third of patients were unable to tolerate single chamber ventricular pacing. These patients could not be identified by simple haemodynamic measurements or clinical characteristics prepacing and would have required upgrading to a dual chamber system. Other important variables such as quality of life and cognitive function were not assessed in the present series. Studies to evaluate the benefits of dual chamber pacing on these variables in patients with carotid sinus syndrome are in progress and may further justify the use of complex systems in older individuals.

\section{CONCLUSION}

Elderly patients with carotid sinus syndrome are likely to develop symptomatic hypotension following single chamber ventricular pacing. This is largely pacemaker induced (although upright vasodepression, which could not be measured in this study, may also contribute). The optimum mode of pacing for individual patients cannot be predicted by simple cardiovascular tests prepacing.

Dr McIntosh was in receipt of a grant from Research into Ageing.

1 Strasberg B, Sagie A, Erdman S, Kusniec J, Sclarovsky S Agmon J. Carotid sinus hypersensitivity and the carotid sinus syndrome. Prog Cardiovasc Dis 1989;31:379-91.

2 Franke H. Uber das Karotissinus-Syndrom und den sogenannten hyperaktiven Karotissinus-Reflex. Stuttgart: Friedrichnten hyperaktiven Karotissinus-Reflex.
Karl Schattauer-Verlag, 1963:149.

3 Thomas JE. Diseases of the carotid sinus-syncope. In: Thomas JE. Diseases of the carotid sinus-syncope. In:
Vinken PJ, Bruyn GW, ed. Handbook of clinical neurology, Vinken PJ, Bruyn GW, ed. Handbook of clinical neurology, vol 11 .

4 Morley CA, Sutton R. Carotid sinus syncope [editorial]. Int f Cardiol 1984;6:287-93.

5 Brignole M, Menozzi C, Lolli G, Bottoni N, Gaggioli G Long-term outcome of paced and nonpaced patients with severe carotid sinus syndrome. Am f Cardiol 1992 69:1039-43.

6 Morley CA, Perrins EJ, Grant P, Chan SL, McBrien DJ, Sutton R. Carotid sinus syncope treated by pacing. Analysis of persistent symptoms and role of atrioventricular sequential pacing. Br Heart f 1982;47:411-18.

7 Travill CM, Sutton R. Pacemaker syndrome: a iatrogenic condition. Br Hear $\mathcal{f}$ 1992;68:163-6.

8 Madigan NP, Flaker GC, Curtis J, Reid J, Mueller KJ Murphy TJ. Carotid sinus hypersensitivity: beneficial effects of dual-chamber pacing. Am $₹$ Cardiol 1984;53: $1034-40$.

9 Rediker DE, Eagle KA, Homma S, Gillam LD, Harthorne JW. Clinical and hemodynamic comparison of VVI versus DDD pacing in patients with DDD pacemakers. $A m^{f}$ Cardiol 1988;61:323-9.

10 Ray SG, Griffith MJ, Jamieson S, Bexton RS, Gold RG. Impact of the recommendations of the British Pacing and 
Electrophysiology Group on pacemaker prescription and on the immediate costs of pacing in the Northern Region. Br Heart F 1992;68:531-4.

11 Report of a working party of the British Pacing and Electrophysiology Group. Recommendations for pacemaker prescription for symptomatic bradycardia. $\mathrm{Br}$ Heart $\mathcal{F}$ 1991;66:185-91.

12 de Belder MA, Linker NJ, Jones S, Camm AJ, Ward DE Cost implications of the British Pacing and Electrophysiology Group's recommendations for pacing. $B M \mathcal{F}$ 1992;305:861-5.

13 McIntosh S, da Costa D, Kenny RA. Benefits of an integrated approach to the investigation of dizziness, falls and syncope in elderly patients referred to a syncope clinic. Age Ageing 1993;22:53-8.

14 Dey AB, Bexton RS, Tyman MM, Charles RG, Kenny RA The impact of a dedicated "Syncope and falls" clinic on pacing practice in northeastern England. PACE 1997;20: pacing prac

15 Kenny RA, Traynor G. Carotid sinus syndrome-clinical characteristics in elderly patients. Age Ageing 1991;20:
449-54.

16 Lakatta EG, Mitchell JH, Pomerance A, Rowe GG Human aging: changes in structure and function. $\mathcal{f} A m$ Coll Cardiol 1987;10:42-7A.

17 Channon KM, Hargreaves MR, Cripps TR, Gardner M Ormerod OJM. DDD vs VVS pacing in patients aged over 75 years with complete heart block: a double-blind crossover comparison. Qf $\mathrm{Fed}$ 1994;87:245-51.

18 British Pacing and Electrophysiology Group Report 1994.

19 Jillings A. Complex, dual chamber, rate-response pacemaker literature review. A report by the Yorkshire Collaborating Centre for Health Services Research Nuffield Institute for Health 1994

20 Brignole M, Sartore B, Barra M, Menozzi C, Lolli G. Is DDD superior to VVI pacing in mixed carotid sinus syndrome? An acute and medium-term study. PACE 1988; 11:1902-10.

21 Brignole M, Menozzi C, Lolli G, Oddone D, Gianfranch L, Bertulla A. Validation of a method for choice of pacing $\mathrm{L}$, Bertulla $\mathrm{A}$. Validation of a method for choice of pacing bradycardia. PACE 1991;14:196-203. 